Article

\title{
Effect of Oxygen Vacancy Ratio on a GaZTO Solar-Blind Photodetector
}

\author{
Jhih-Chun Syu ${ }^{1}$, Ming-Hung Hsu ${ }^{1}$, Sheng-Po Chang ${ }^{1, * \mathbb{B}}$, Shoou-Jinn Chang ${ }^{1}$ and Lucent Lu ${ }^{2}$ \\ 1 Institute of Microelectronics \& Department of Electrical Engineering, Center for Micro/Nano Science and \\ Technology, Advanced Optoelectronic Technology Center, National Cheng Kung University, \\ Tainan 70101, Taiwan; q16054011@mail.ncku.edu.tw (J.-C.S.); hsuminghung0121@gmail.com (M.-H.H.); \\ changsj@mail.ncku.edu.tw (S.-J.C.) \\ 2 SungShine Optelectronics Co., Ltd., Shenzhen 518067, China; lucent@ssotgd.com \\ * Correspondence: changsp@mail.ncku.edu.tw; Tel.: +886-6-275-7575 (ext. 62400-1208)
}

Received: 20 June 2018; Accepted: 20 August 2018; Published: 21 August 2018

\begin{abstract}
A gallium-zinc-tin-oxide (GaZTO) solar-blind photodetector was fabricated via radio frequency sputtering. The transmittance of the GaZTO thin film was $>80 \%$ in the visible light region, and its energy bandgap ranged from 4.11 to $4.23 \mathrm{eV}$. Manipulating the oxygen flows changed the ratio of oxygen vacancies, which was confirmed by X-ray photoelectron spectroscopy. The ratio of oxygen vacancies in the GaZTO thin films impacted the performance of the photodetectors. The photocurrent, responsivity, and ultraviolet-visible rejection ratio of the GaZTO solar-blind photodetector were $1.23 \times 10^{-6} \mathrm{~A}, 9.12 \times 10^{-2} \mathrm{~A} / \mathrm{W}$, and $3.48 \times 10^{4}$, respectively, at a $10 \mathrm{~V}$ bias under $280 \mathrm{~nm}$ light illumination. The transient responses of the synthesized photodetectors were measured and discussed.
\end{abstract}

Keywords: GaZTO; RF-sputter; solar-blind photodetector

\section{Introduction}

Currently, photodetectors are utilized in many fields, such as pollution detection, flame detection, space communication, light-wave communication, and missile-launch detection [1-3]. Ultraviolet (UV) light is categorized into UV-A, UV-B, and UV-C, whose wavelength ranges are $320-400 \mathrm{~nm}$, 280-320 nm, and 200-280 nm, respectively. Photodetectors sensitive to UV-A and UV-B light are called visible-blind photodetectors. A solar-blind photodetector is specifically sensitive to UV-C radiation. As the detection of UV light requires the assistance of wide-bandgap materials, scientists endeavor to find suitable materials that can be applied for UV light detection.

The semiconducting materials commonly used to detect UV-C light are $\mathrm{MgZnO}$ [4], $\mathrm{Ga}_{2} \mathrm{O}_{3}$ [5], and AlGaN [6]. The bandgaps of $\mathrm{MgZnO}$ and AlGaN are 3.3-7.8 and 3.44-6.28 eV, respectively, and can be tuned by adjusting the chemical content of the materials [4,7]. Owing to its high transmittance in the visible light region and wide-bandgap nature, $\mathrm{ZnO}$ has been employed for many photoelectronic components, such as solar cells, light-emitting diodes, and UV photodetectors [8-10]. According to Hosono's previous work, a metal cation whose electronic configuration is $(n-1) d^{10} n s^{0}$, where $n \geq 5$, can improve the carrier mobility [11]. Due to the ns spherical orbitals of a heavy-metal cation, the adjacent ns orbitals overlap on the amorphous nature, and carriers in the amorphous oxide semiconductor move more smoothly than those in amorphous silicon, whose electronic configuration is $s p^{3}$ [11]. Accordingly, numerous amorphous oxides containing heavy metals, such as Tin (Sn) and indium (In), have drawn attention and scientists are showing a tendency of doping suitable metals into $\mathrm{ZnO}$. Selecting $\mathrm{Sn}$ as the dopant is expected to enhance the mobility of $\mathrm{ZnO}$-based components because of its desirable ionic electronic configuration $\left([\mathrm{Kr}] 4 d^{10}\right)$. Zinc tin oxide (ZTO) is an attractive 
photo-sensing material. With its bandgap of 3.37-3.93 eV, it is able to detect UV-B, UV-C, and part of UV-A radiation. To allow for the detection of only UV-B and UV-C light, gallium is doped into $\mathrm{ZTO}$ to form the quaternary metal oxide GaZTO. Carriers of $\mathrm{ZnO}$, originating from oxygen vacancies and zinc interstitials, can be suppressed by appropriately doping metal elements to alter the device performance [11,12]. Gallium is an alternative candidate as a suppressor of oxygen vacancies. GaZTO is a novel material that is still seldom studied in academia. It is expected that the bandgap of GaZTO can be engineered by changing the composition of gallium, zinc, and tin [13].

In this study, we fabricated GaZTO solar-blind photodetectors using a radiofrequency (RF)-sputtering system. The optical characteristics, chemical-bonding states, and electrical performances of the devices were investigated in detail.

\section{Materials and Methods}

The structure of the GaZTO solar-blind photodetector is shown in Figure 1. Quartz substrates were cleaned using acetone, isopropyl alcohol, and deionized water sequentially with an ultrasonicator and then dried with a nitrogen flow. A $100 \mathrm{~nm}$ GaZTO thin film was grown on the substrate using an RF-sputtering system (KD-SPUTTER, Kao Duen Technology Corp., New Taipei City, Taiwan) with a single GaZTO target (molar ratio of Ga:Zn:Sn = 1:1:1). The RF power was $80 \mathrm{~W}$, and the working pressure was $5 \mathrm{mTorr}$. The sputtering was performed in argon/oxygen gas mixtures. Herein, we denote thin films deposited at argon-to-oxygen flow ratios of 50:0, 48:2, and 45:5 as Samples A, B, and $\mathrm{C}$, respectively. After the thin-film deposition, the samples were annealed at $300{ }^{\circ} \mathrm{C}$ for $1 \mathrm{~h}$ in a furnace. Finally, nickel/gold $(30 \mathrm{~nm} / 70 \mathrm{~nm})$ was thermally deposited to serve as the interdigitated electrodes (by ULVAC thermal evaporation system, ULVAC, Methuen, MA, USA), which were defined by the shadow mask. The finger width and the finger spacing were 100 and $200 \mu \mathrm{m}$, respectively. $I-V$ curves were measured in the dark at room temperature using an Agilent B1500 semiconductor parameter analyzer (Agilent Technologies, Santa Clara, CA, USA). The illumination was generated from a $250 \mathrm{~W}$ Xe lamp via a monochromator (H10 UV, HORIBA Jobin Yvon, Edison, NJ, USA) to create the monochromatic light. The illumination area of a photodetector was $2200 \mu \mathrm{m} \times 2000 \mu \mathrm{m}$. To observe the film transparency and determine the elemental compositions, three types (Samples A, $\mathrm{B}$, and C) of $100 \mathrm{~nm} \mathrm{GaZTO}$ thin films were prepared via the same process, without the patterning of the metals. The transmittance and absorption spectra were recorded using a UV-3101 UV-Visible (Vis)-near-infrared spectrophotometer (Shimadzu UV-3101PC, SHIMADZU Corp., Kyoto, Japan), and X-ray photoelectron spectroscopy (XPS) (VG ESCALAB220i-XL, Thermo Scientific, Waltham, MA, USA) was performed to analyze the chemical-bonding state of oxygen.

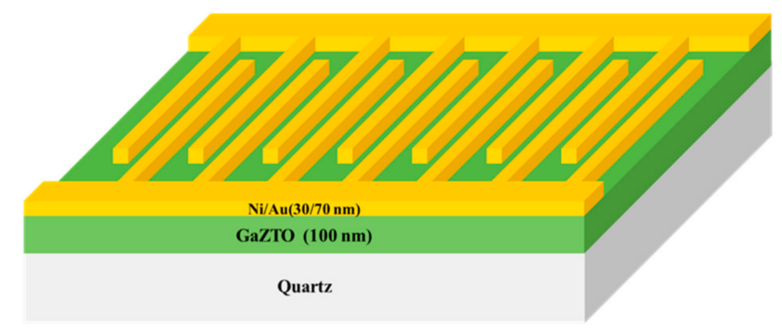

Figure 1. Structural diagram of the GaZTO solar-blind photodetector.

\section{Results and Discussion}

Figure 2a shows the transmittance of the GaZTO thin films (Samples A, B, and C). All films clearly showed a transmittance of $>80 \%$ in the visible light region. To extract the optical bandgap of the GaZTO thin film, the relationship between the absorption coefficient and the incident photon energy 
was plotted, as shown in Figure 2b. The bandgaps were determined according to Tauc's Law [14], as follows:

$$
(\alpha h v)^{2}=B\left(h v-E_{\mathrm{g}}\right)
$$

where $\alpha$ is the absorption coefficient, $h$ is Planck's constant, $v$ is the frequency of incident light, $B$ is a constant, and $E_{\mathrm{g}}$ is the bandgap of the material. By extrapolating the linear part of the curve to intersect with the $x$-axis, the bandgap was determined to be $4.11-4.23 \mathrm{eV}$ and found to increase with the decreasing oxygen flow. The transmittance decreases dramatically when the light wavelength is $<300 \mathrm{~nm}$, which corresponds to the calculated bandgap values. All of the GaZTO thin films exhibit a relatively high bandgap energy and desirable transmittance, which are beneficial for optoelectronic applications.
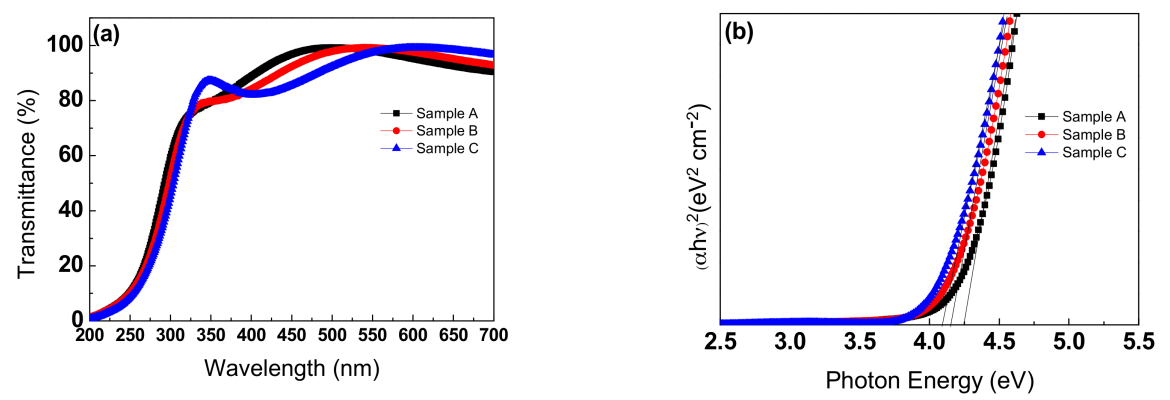

Figure 2. (a) Transmittance spectrum of Samples A, B, and C. (b) absorption coefficient versus photon energy for these three samples.

The decrease of the optical bandgap of the GaZTO thin film with the increasing oxygen flow ratio results from the variation of the elemental composition of GaZTO. As shown in Table 1, with the increase of the oxygen flow ratio, the atomic ratio of $\mathrm{Zn}$ increases, and those of $\mathrm{Ga}$ and $\mathrm{Sn}$ decrease. It is possible that the deposition rate of $\mathrm{ZnO}$ increases while those of $\mathrm{Ga}_{2} \mathrm{O}_{3}$ and $\mathrm{SnO}_{2}$ decrease with the increasing oxygen flow ratio [15]. It is known that the bandgap of $\mathrm{ZnO}(3.3 \mathrm{eV})$ is lower than those of $\mathrm{Ga}_{2} \mathrm{O}_{3}(4.99 \mathrm{eV})$ and $\mathrm{SnO}_{2}(3.93 \mathrm{eV})$; thus, the bandgap of the GaZTO thin film decreased with the increasing oxygen flow ratio.

Table 1. Atomic composition of the GaZTO thin film with different oxygen flow ratios, confirmed via X-ray photoelectron spectroscopy (XPS).

\begin{tabular}{ccccc}
\hline Sample & Zn (\%) & Ga (\%) & Sn (\%) & O (\%) \\
\hline A & 6.3 & 19.9 & 23.0 & 50.7 \\
B & 9.6 & 19.3 & 14.8 & 56.4 \\
C & 10.1 & 18.4 & 15.2 & 56.4 \\
\hline
\end{tabular}

The number of oxygen vacancies in a ZnO-based material significantly affects the carrier concentration [11]. The XPS O 1s spectra of Samples A, B, and C-which are presented in Figure 3-were used to analyze the variation in the quantity of oxygen vacancies. The raw data curve can be deconvoluted into three peaks via Gaussian fitting, which are assigned to metal-oxygen bonding $\left(\mathrm{O}_{\text {met }}\right.$, centered at $\left.530.6 \mathrm{eV}\right)$, oxygen vacancies $\left(\mathrm{O}_{\mathrm{vac}}\right.$, centered at $\left.531.1 \mathrm{eV}\right)$, and bonding with a hydroxyl group or the $\mathrm{H}_{2} \mathrm{O}$ from the surface of the thin film $\left(\mathrm{O}_{\text {surf }}\right.$, centered at $\left.532.1 \mathrm{eV}\right)$ [16]. The ratios of $\mathrm{O}_{\text {met }}, \mathrm{O}_{\mathrm{vac}}$, and $\mathrm{O}_{\text {surf }}$ for each sample are listed in Table 2. Notably, the ratio of oxygen vacancies decreases from $23.4 \%$ to $20.0 \%$ with the increasing oxygen flow ratio. That is, the relative amount of oxygen vacancies is reduced when the oxygen flow ratio is increased. This is an important issue for the device performance, which is discussed later. 
Figure 4 plots the photocurrents and dark currents of GaZTO photodetectors with different oxygen flow ratios. The wavelength of light irradiation was $280 \mathrm{~nm}$. The fabricated photodetectors were subject to light illumination with different wavelengths for 1 min each time and then immediately probed for $I-V$ measurement to ascertain their performances. For all samples, the order of magnitude of the dark currents is -11 , indicating that the GaZTO devices have acceptable turn-off characteristics. When the devices are measured in the dark, the oxygen molecules in the air are absorbed by the GaZTO thin film. The absorbed oxygen molecules capture the free electrons in the thin film, contributing to the generation of a depletion region near the film surface [3]. Hence, the dark current is suppressed. The photocurrents of Samples A, B, and C at $10 \mathrm{~V}$ are summarized in Table 3. The photocurrent gradually decreased because the quantity of carriers was reduced. Owing to the increased amount of photo-generated carriers due to oxygen vacancies under light illumination, the photocurrent was enhanced with the decreasing oxygen flow ratio [17].
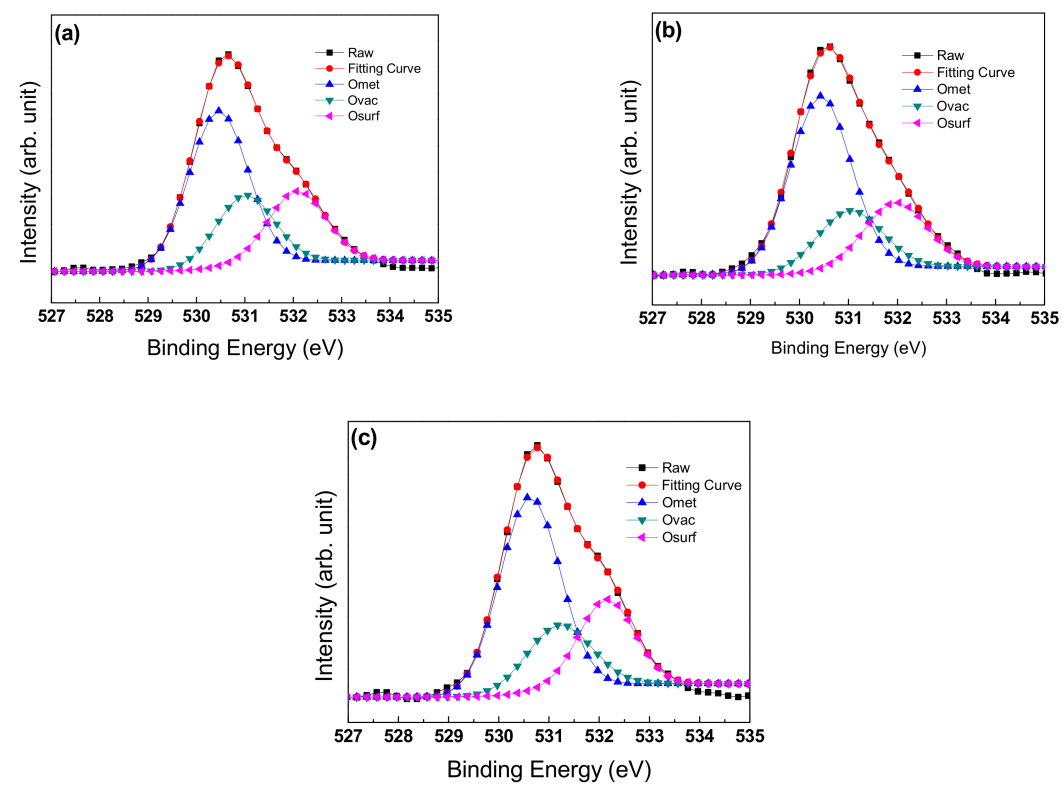

Figure 3. XPS O 1s spectra of GaZTO thin films with different experimental conditions: (a) Sample A; (b) Sample B; and (c) Sample C.

Table 2. Relative peak area ratio of the GaZTO thin films.

\begin{tabular}{cccc}
\hline Sample & $\mathbf{O}_{\text {met }}(\mathbf{\%})$ & $\mathbf{O}_{\text {vac }}(\mathbf{\%})$ & $\mathbf{O}_{\text {surf }} \mathbf{( \% )}$ \\
\hline $\mathrm{A}$ & 53.3 & 23.4 & 23.3 \\
$\mathrm{~B}$ & 57.6 & 20.5 & 21.9 \\
$\mathrm{C}$ & 56.9 & 20.0 & 23.1 \\
\hline
\end{tabular}

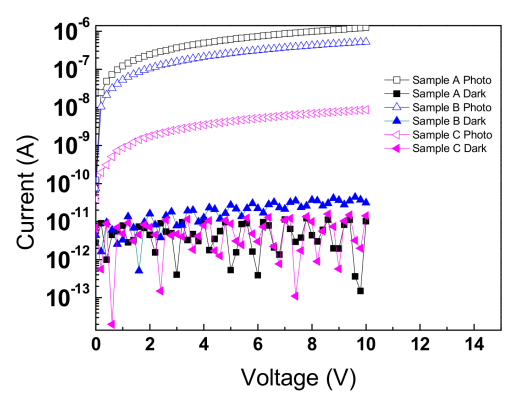

Figure 4. $I-V$ characteristics of the three GaZTO photodetectors measured in the dark and under $280 \mathrm{~nm}$ UV light. 
The responsivity of the GaZTO photodetectors as a function of the wavelength is illustrated in Figure 5. Responsivity $(R)$ is one of the crucial parameters for quantifying the performance of a photodetector. It is defined as the generated photocurrent per unit of incident UV light power, as follows:

$$
R=\left(I_{\text {photo }}-I_{\text {dark }}\right) / P_{\text {photo }}
$$

where $I_{\text {photo }}$ is the photocurrent, $I_{\text {dark }}$ is the dark current, and $P_{\text {photo }}$ is the power of the incident light. With a $10 \mathrm{~V}$ bias, the responsivity of Samples A, B, and C is $9.12 \times 10^{-2}, 3.9 \times 10^{-2}, 6.4 \times 10^{-4} \mathrm{~A} / \mathrm{W}$, respectively, at $280 \mathrm{~nm}$. Notably, when the wavelength is $<280 \mathrm{~nm}$, the responsivity is higher than those at other wavelengths in the visible light region. This suggests that our GaZTO photodetectors are applicable for solar-blind detection. We also employed the UV-V is rejection ratio to determine whether a photodetector can more effectively reject the interference of visible light. This ratio is defined as the responsivity at $280 \mathrm{~nm}$ divided by the responsivity at $450 \mathrm{~nm}$. Table 3 summarizes the responsivity and rejection ratios of the GaZTO photodetectors. Sample B exhibits the highest rejection ratio, indicating that it can distinguish UV light from visible light more effectively than the other two samples.

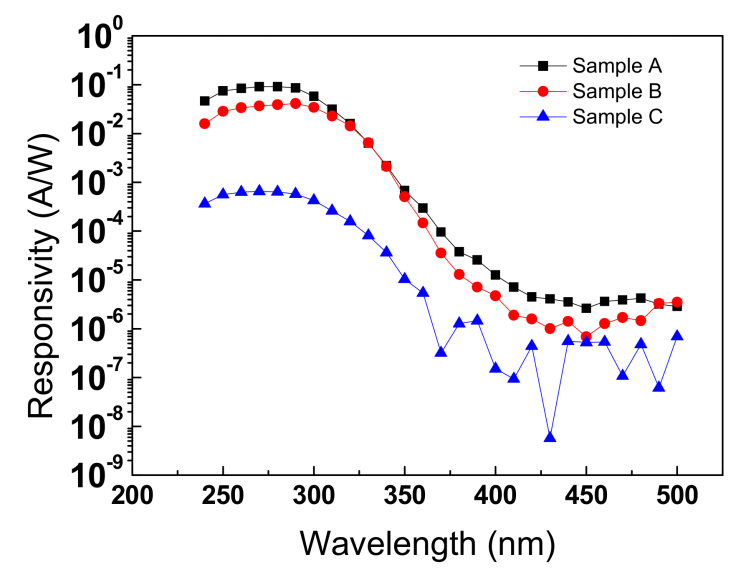

Figure 5. Responsivity of GaZTO photodetectors with different oxygen flow ratios.

Table 3. Photocurrent, responsivity, rejection ratio, rise time, and fall time of the GaZTO photodetectors with different oxygen flow ratios.

\begin{tabular}{cccccc}
\hline Sample & $\begin{array}{c}\text { Photocurrent } \\
\text { at } \mathbf{1 0 ~ V ~ ( A ) ~}\end{array}$ & $\begin{array}{c}\text { Responsivity at } \\
\mathbf{2 8 0} \mathbf{~ n m} \mathbf{( A / W )}\end{array}$ & $\begin{array}{c}\text { Rejection } \\
\text { Ratio }\end{array}$ & $\begin{array}{c}\text { Growth Time } \\
\text { Constant (s) }\end{array}$ & $\begin{array}{c}\text { Decay Time } \\
\text { Constant (s) }\end{array}$ \\
\hline $\mathrm{A}$ & $1.23 \times 10^{-6}$ & $9.12 \times 10^{-2}$ & $3.48 \times 10^{4}$ & 125.8 & 30.4 \\
$\mathrm{~B}$ & $5.26 \times 10^{-7}$ & $3.9 \times 10^{-2}$ & $5.65 \times 10^{4}$ & 77.8 & 9.6 \\
$\mathrm{C}$ & $8.63 \times 10^{-9}$ & $6.4 \times 10^{-4}$ & $1.14 \times 10^{3}$ & 66.1 & 11.9 \\
\hline
\end{tabular}

Figure 6 shows the transient characteristics of each sample under periodic UV illumination of $280 \mathrm{~nm}$ at a $10 \mathrm{~V}$ bias. The turn-on time intervals for Samples A, B, and C were 450, 250, and $250 \mathrm{~s}$, respectively. The turn-off time intervals were 450,100, and $150 \mathrm{~s}$, respectively. The transient responses are reproducible. The exponential relaxation model can be used for the switching time measurement. Curve fitting was implemented to the first switching period of both Samples A and B, and to the third switching period of Sample C. The growth time/decay time constants can be extracted from the model, and the two constants are used to evaluate the performance of the switching characteristics of devices [18]. The growth/decay time constants of the three samples are listed in Table 3. Sample C has better transient characteristics; it is faster. The growth/decay time constants of Sample $\mathrm{C}$ are 66.1 and 11.9 s, respectively. Although the constants of Sample C are relatively desirable, Sample C 
demonstrated an unstable behavior upon UV light illumination, which is considered to be related to oxygen flow ratio of $10 \%$ and inferior thin film electrical performance.
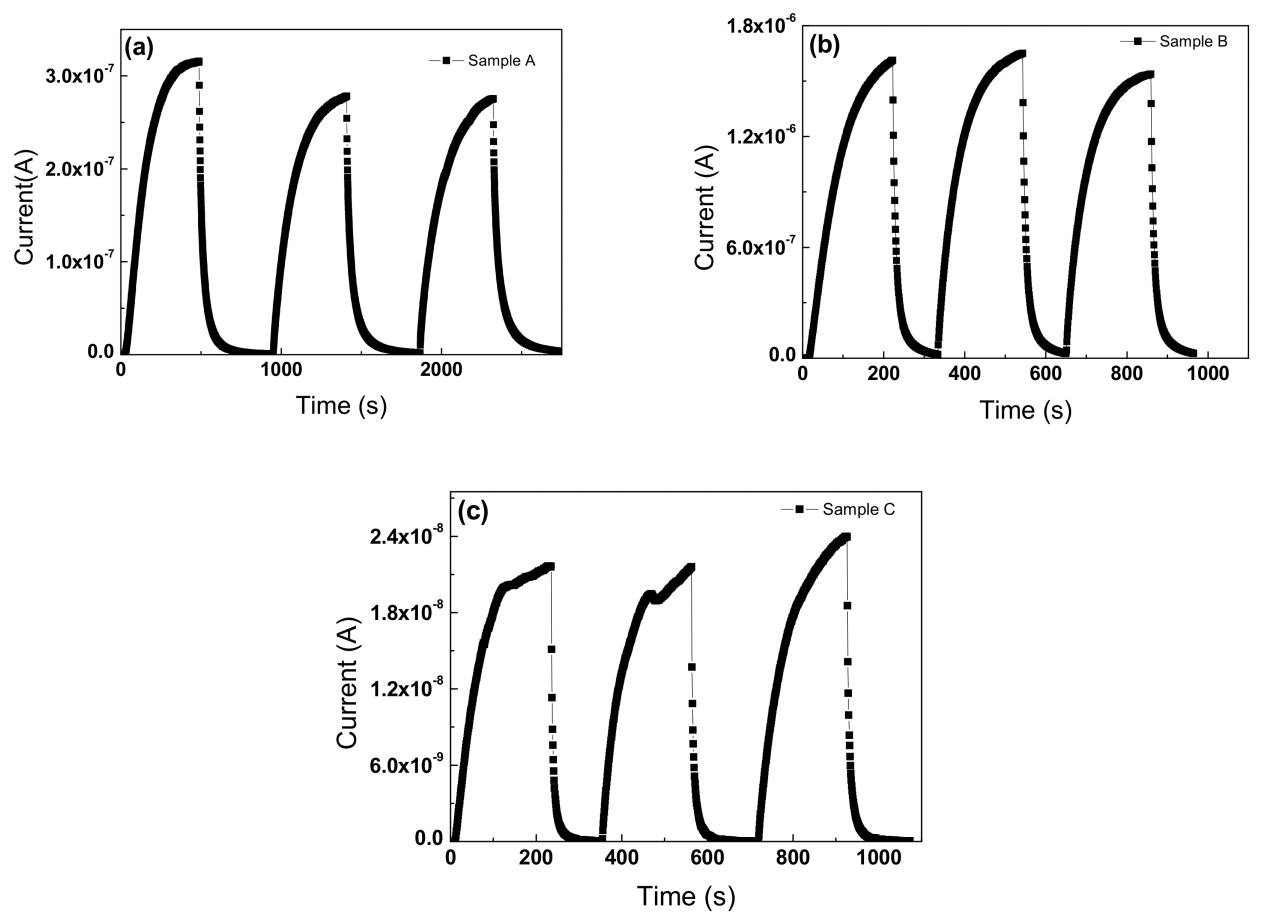

Figure 6. Time responses of (a) Sample A; (b) Sample B; and (c) Sample C.

Tables 2 and 3 reveal that a high relative amount of oxygen vacancies leads to a high photocurrent, which is attributed to the photo-generated carriers induced by light irradiation [17]. Oxygen vacancies can induce an increase in the photocurrent, but they are a type of defect and influence the device performance to some extent. Increasing the ratio of oxygen vacancies leads to the deterioration of the rise time and fall time, as shown in Tables 2 and 3. The photoexcited oxygen vacancies capture carriers, which is time-consuming [17]. As a result, if the ratio of oxygen vacancies increases, the rise and fall times increase. Thus, there is a tradeoff between the response time and the photocurrent. In addition, it is known that passivation can ameliorate the performance of an electronic device. Even though our devices do not have passivation, Samples A and B still performed very well and functionally. Achievement of advanced device performance by passivating devices will be our next issue to be investigated. Table 4 lists previous works focusing on solar-blind photodetectors. Compared with these photodetectors, Sample A exhibits a higher responsivity than the devices synthesized with other materials, revealing that GaZTO is a promising candidate for solar-blind detection.

Table 4. Responsivity of photodetectors fabricated with different materials using various methods. It is noted that RF-MBE stands for radio-frequency plasma assisted molecular beam epitaxy, MOCVD for metal-organic chemical vapor deposition, L-MBE for laser molecular beam epitaxy, and VLS for vapor-liquid-solid technique.

\begin{tabular}{ccccc}
\hline Material & Method & Responsivity $(\mathbf{m A} / \mathbf{W})$ & Incident Wavelength $(\mathbf{n m})$ & Ref. \\
\hline $\mathrm{Mg}_{0.55} \mathrm{Zn}_{0.45} \mathrm{O}$ & $\mathrm{RF}-\mathrm{MBE}$ & 22 & 270 & {$[19]$} \\
$\mathrm{Mg}_{0.48} \mathrm{Zn}_{0.52} \mathrm{O}$ & $\mathrm{MOCVD}$ & 16 & 283 & {$[20]$} \\
$\mathrm{Sn} \mathrm{Doped} \beta-\mathrm{Ga}_{2} \mathrm{O}_{3}$ & $\mathrm{~L}-\mathrm{MBE}$ & 36 & 254 & {$[21]$} \\
$\beta-\mathrm{Ga}_{2} \mathrm{O}_{3}$ & VLS & 0.372 & 255 & {$[22]$} \\
$\mathrm{BeMgZnO}$ & $\mathrm{RF}-\mathrm{MBE}$ & 18 & 241 & {$[23]$} \\
$\mathrm{GaZTO}$ & RF-sputter & 91.2 & 280 & this study \\
\hline
\end{tabular}




\section{Conclusions}

We proposed a simple, cost-effective approach to fabricate solar-blind photodetectors with a GaZTO sensing layer using an RF-sputtering system. The optical characteristics were measured to ascertain the bandgap and transmittance. The energy bandgap of the GaZTO thin film was 4.11-4.23 eV, indicating that the film can be applied for solar-blind detection. Adjusting the oxygen flow ratio during sputtering resulted in the variation of the number of oxygen vacancies, which is a key factor influencing the device performance. The photocurrent, responsivity, rejection ratio, and time response were measured in this study. The sample with an oxygen ratio of $4 \%$ exhibited excellent performance, with a photocurrent of $1.23 \times 10^{-6} \mathrm{~A}$ and responsivity of $9.12 \times 10^{-2} \mathrm{~A} / \mathrm{W}$. In the transient-response measurement, the sample with an oxygen ratio of $10 \%$ exhibited the shortest rise and fall times. Furthermore, it was discovered that varying the ratio of oxygen vacancies could enhance the performance of the GaZTO photodetectors. The responsivity of the sample with an oxygen ratio of $4 \%$ exceeded that of previously reported devices.

Author Contributions: Conceptualization, S.-P.C.; Formal Analysis, J.-C.S. and M.-H.H.; Funding Acquisition, L.L.; Investigation, J.-C.S., M.-H.H. and S.-P.C.; Project Administration, S.-P.C. and S.-J.C.; Resources, S.-J.C.; Supervision, L.L.; Writing-Original Draft, J.-C.S. and M.-H.H.

Funding: This work was funded by the Ministry of Science and Technology, Taiwan (MOST 106-2221-E-006-178 and MOST 106-2221-E-006-172-MY3).

Acknowledgments: The authors thanked the Center for Frontier Materials and Micro/Nano Science and Technology, National Cheng Kung University, Taiwan; and the Advanced Optoelectronic Technology Center, National Cheng Kung University, for projects from the Ministry of Education.

Conflicts of Interest: The authors declare no conflict of interest.

\section{References}

1. Hsu, M.-H.; Chang, S.-P.; Chang, S.-J.; Wu, W.-T.; Li, J.-Y. Influence of oxygen on the performance of indium titanium zinc oxide UV sensors fabricated via RF sputtering. Mater. Sci. Semicond. Process. 2018, 74, 297-302. [CrossRef]

2. Zhang, Y.; Wang, J.; Zhu, H.; Li, H.; Jiang, L.; Shu, C.; Hu, W.; Wang, C. High performance ultraviolet photodetectors based on an individual $\mathrm{Zn}_{2} \mathrm{SnO}_{4}$ single crystalline nanowire. J. Mater. Chem. 2010, 20, 9858-9860. [CrossRef]

3. Wang, W.; Pan, X.; Dai, W.; Zeng, Y.; Ye, Z. Ultrahigh sensitivity in the amorphous ZnSnO UV photodetector. RSC Adv. 2016, 6, 32715-32720. [CrossRef]

4. Xie, X.; Zhang, Z.; Liu, B.; Wang, S.; Jiang, M.; Shan, C.; Zhao, D.; Chen, H.; Shen, D. Enhanced solar-blind responsivity of photodetectors based on cubic $\mathrm{MgZnO}$ films via gallium doping. Opt. Express 2014, 22, 246-253. [CrossRef] [PubMed]

5. Feng, W.; Wang, X.; Zhang, J.; Wang, L.; Zheng, W.; Hu, P.; Cao, W.; Yang, B. Synthesis of two-dimensional $\beta-\mathrm{Ga}_{2} \mathrm{O}_{3}$ nanosheets for high-performance solar blind photodetectors. J. Mater. Chem. C 2014, 2, 3254-3259. [CrossRef]

6. Brendel, M.; Helbling, M.; Knigge, A.; Brunner, F.; Weyers, M. Solar-blind AlGaN MSM photodetectors with $24 \%$ external quantum efficiency at 0 V. Electron. Lett. 2015, 51, 1598-1599. [CrossRef]

7. Ronaldo, R.P.; Clóvis, C.; Marcelo, M.; Luiz, G.F.; Jürgen, F.; Lara, K.T. Accurate band gaps of AlGaN, InGaN, and AlInN alloys calculations based on LDA-1/2 approach. Appl. Phys. Lett. 2011, 98, 151907.

8. Zhang, X.; Huang, X.; Li, C.; Jiang, H. Dye-sensitized solar cell with energy storage function through PVDF/ZnO nanocomposite counter electrode. Adv. Mater. 2013, 25, 4093-4096. [CrossRef] [PubMed]

9. Abdelfatah, M.; Ismail, W.; El-Shaer, A. Low cost inorganic white light emitting diode based on submicron $\mathrm{ZnO}$ rod arrays and electrodeposited $\mathrm{Cu}_{2} \mathrm{O}$ thin film. Mater. Sci. Semicond. Process 2018, 81, 44-47. [CrossRef]

10. Dong, M.; Wang, Y.; Li, Z.; Weng, Z.; Yu, N. Simple fabrication of homogeneous ZnO core/shell nanorod arrays for ultraviolet photodetectors. J. Nanosci. Nanotechnol. 2018, 18, 5686-5691. [CrossRef] [PubMed]

11. Hosono, H. Ionic amorphous oxide semiconductors: Material design, carrier transport, and device application. J. Non-Cryst. Solids 2006, 352, 851-858. [CrossRef] 
12. Zhang, S.B.; Wei, S.-H.; Zunger, A. Intrinsic $n$-type versus $p$-type doping asymmetry and the defect physics of ZnO. Phys. Rev. B 2001, 63, 075205. [CrossRef]

13. Kim, H.-R.; Park, J.; Lee, S.-H.; Lee, G.-H.; Song, P.-G.; Kang, Y.-C.; Kim, D.-H. Effects of Ga concentration on electrical and physical properties of amorphous $\mathrm{Ga}-\mathrm{Zn}-\mathrm{Sn}-\mathrm{O}$ semiconductor thin films. Electrochem. Solid State Lett. 2011, 14, H411-H414. [CrossRef]

14. Jauc, T.; Grigorovici, R.; Vancu, A. Optical properties and electronic structure of amorphous germanium. Phys. Status Solidi B 1966, 15, 627-637.

15. Kim, D.-H.; Kim, H.-R.; Kwon, J.-D.; Lee, G.-H.; Lee, H.S.; Im, S. Sputter-deposited Ga-Sn-Zn-O thin films for transparent thin film transistors. Phys. Status Solidi A 2011, 208, 2934-2938. [CrossRef]

16. Ahn, B.D.; Jeon, H.J.; Park, J.-S. Effects of Ga:N Addition on the electrical performance of zinc tin oxide thin film transistor by solution-processing. ACS Appl. Mater. Interfaces 2014, 6, 9228-9235. [CrossRef] [PubMed]

17. Wu, P.; Zhang, J.; Lu, J.; Li, X.; Wu, C.; Sun, R.; Feng, L.; Jiang, Q.; Lu, B.; Pan, X.; et al. Instability induced by ultraviolet light in $\mathrm{ZnO}$ thin-film transistors. IEEE Trans. Electron Devices 2014, 61, 1431-1435.

18. Liu, N.; Fang, G.; Zeng, W.; Zhou, H.; Cheng, F.; Zheng, Q.; Yuan, L.; Zou, X.; Zhao, X. Direct growth of lateral ZnO nanorod UV photodetectors with Schottky contact by a single-step hydrothermal reaction. ACS Appl. Mater. Interfaces 2010, 2, 1973-1979. [CrossRef]

19. Hou, Y.; Mei, Z.; Liu, Z.; Zhang, T.; Du, X. $\mathrm{Mg}_{0.55} \mathrm{Zn}_{0.45} \mathrm{O}$ solar-blind ultraviolet detector with high photoresponse performance and large internal gain. Appl. Phys. Lett. 2011, 98, 103506. [CrossRef]

20. Wang, L.; Ju, Z.; Shan, C.; Zheng, J.; Shen, D.; Yao, B.; Zhao, D.; Zhang, Z.; Li, B.; Zhang, J. MgZnO metal-semiconductor-metal structured solar-blind photodetector with fast response. Solid State Commun. 2009, 149, 2021-2023. [CrossRef]

21. Zhao, X.; Cui, W.; Wu, Z.; Guo, D.; Li, P.; An, Y.; Li, L.; Tang, W. Growth and characterization of Sn doped $\beta-\mathrm{Ga}_{2} \mathrm{O}_{3}$ thin films and enhanced performance in a solar-blind photodetector. J. Electron. Mater. 2017, 46, 2366-2372. [CrossRef]

22. Weng, W.Y.; Hsueh, T.J.; Chang, S.-J.; Huang, G.J.; Hung, S.C. Growth of $\mathrm{Ga}_{2} \mathrm{O}_{3} \mathrm{Nanowires}$ and the Fabrication of Solar-Blind Photodetector. IEEE Trans. Nanotechnol. 2011, 10, 1047-1052. [CrossRef]

23. Su, L.; Zhu, Y.; Yong, D.; Chen, M.; Ji, X.; Su, Y.; Gui, X.; Pan, B.; Xiang, R.; Tang, Z. Wide range bandgap modulation based on $\mathrm{ZnO}$-based alloys and fabrication of solar blind $\mathrm{UV}$ detectors with high rejection ratio. ACS Appl. Mater. Interfaces 2014, 6, 14152-14158. [CrossRef] [PubMed] 